\title{
Adequacy of equipment available for the insertion of chest drains in tertiary care units in Sri Lanka: a multi center study
}

\author{
D.V.T. Harischandra ${ }^{1}$, H.V.G. Hewage ${ }^{1}$, L. Amarashriyan ${ }^{2}$, K. Indrapala ${ }^{3}$, N. Perera ${ }^{1}$, J.M.R.G Jayaweera ${ }^{4}$ \\ ${ }^{1}$ Teaching Hospital Karapitiya, Galle, Sri Lanka \\ ${ }^{2}$ Teaching Hospital Mahamodara, Sri Lanka \\ ${ }^{3}$ General hospital Balapitiya, Sri Lanka $\quad{ }^{4}$ General Hospital, Avissawella, Sri Lanka
}

Key words: Chest drain; intercostal tube; adequacy of equipment

\begin{abstract}
Introduction

Insertion of a chest drain is a common, potentially life-saving procedure, and most doctors will be required to insert a chest drain at some stage of their career, regardless of their specialty. Complications can occur during this procedure that can be life threatening. Not having the required equipment at the crucial time has been highlighted as an important cause of iatrogenic complications.
\end{abstract}

\section{Objectives}

Our objectives were to formulate a checklist of items required for the safe insertion of chest drains and to assess the adequacy of the equipment available for the procedure within the tertiary care setting of Sri Lanka based on this checklist.

\section{Methods}

A checklist was compiled based on the British Thoracic Society guidelines with modifications according to the Sri Lankan setting. This was further modified after a consensus from an expert panel using the Delphi technique. This checklist was used to perform a descriptive cross-sectional study within tertiary care units in Sri Lanka selected using a multi-staged sampling technique.

\section{Results}

A checklist was formulated consisting of 10 items in the units and nine items in a chest drain pack. Twenty nine units were assessed from five tertiary care hospitals of five provinces of Sri Lanka: Although 75.9\% units had designated "chest drain insertion packs", the mean availability of instruments inside them were $52.5 \%$. Only $73 \%$ of units had curved instruments to facilitate safe insertion of a drain. Only $7 \%$ of units had

Correspondence: Tolusha Harischandra

E-mail: tolushah@gmail.com

Received: 12-10-2017 Accepted: 08-04-2018

(D) http://orcid.org/0000-0001-9791-5431

DOI: http://doi.org/10.4038/sljs.v36i1.8473

The Sri Lanka Journal of Surgery 2018; 36(1): 1-4 more than one pack. The availability of equipment required to be easily available within the unit was $94.8 \%$. However, only $24 \%$ units used the safer non-trocar chest drain exclusively.

\section{Conclusions}

There is a deficiency of organized instruments and especially non-trocar tubes, even in our tertiary care hospitals. This is likely to make chest drain insertion unsafe in the majority of our units. Availability of a "chest drain checklist" among units could guide the nurses to keep available the required equipment at hand and to set up the trolley at short notice.

\section{Introduction}

Insertion of a chest drain is a common lifesaving procedure that is widely used throughout the surgical, medical, trauma and critical care specialties. It is used to drain either actual or potential pleural air or fluid either as an elective or emergency procedure. Most doctors will insert a chest drain at some stage of their career regardless of their specialty. These may cause iatrogenic complications that can be life threatening or severely debilitating. Of a survey of the hospitals in the United Kingdom (UK), 67\% reported at least one major incident involving chest drain insertion [1].

As chest drain insertion can be an emergency procedure, the required equipment should be organized well ahead and be readily available in adequate quantities. The equipment required for chest drain insertion is relatively simple and inexpensive. However, non-availability of equipment has been highlighted as a cause of iatrogenic complications in UK [2]. Furthermore, the use of chest drains with sharp trocar tips have been found to cause a $6-14 \%$ increase of operator-related complications [3]. British Thoracic Society (BTS) guidelines have listed out the required equipment for the safe insertion of a chest drain and also recommended the use of blunt dissection and the use of chest drains without trocars [4].

It has been our impression that the equipment within hospitals in Sri Lanka too, is less than satisfactory. Often, the proper equipment is not available when it is needed most, leading to unnecessary delays and hassle. In multiple casualties, this situation is worse. For children, often only chest drains of 
adult sizes are available. In most instances sharp-tipped trocars, that can be dangerous in inexperienced hands, are the only chest drains available. Interestingly, chest drains with trocars are more expensive than its safer non-trocar counterpart. Identifying and correcting these inadequacies could ensure a successful outcome of this procedure, preventing unnecessary complications and excessive cost to our health system. Formulating a list of equipment required for chest drain insertion, which to date has not been available in our hospitals, would be useful to guide our staff.

Therefore, the objectives of our study were twofold: i.e. firstly, to formulate a checklist of items for the insertion of chest tubes in the Sri Lankan setting and secondly to assess the adequacy of equipment available for chest drain insertion based on the above checklist in the tertiary care hospitals of Sri Lanka.

\section{Methods}

The study received approval from the Ethical Review Committee of the Faculty of Medicine, University of Ruhuna, Sri Lanka.

A checklist was compiled based on the British Thoracic Society (BTS) guidelines with modifications according to the Sri Lankan setting. Views of a panel of experts consisting of cardiothoracic surgeons, general surgeons and nursing officers were obtained via the Delphi technique and a consensus was taken in finalizing the checklist.

Data collectors were trained by the principal investigator on filling the data form in order to minimize inter-observer bias. A pre-test was carried out at the Cardiothoracic Unit (CTU) at Teaching hospital Karapitiya and adjustments were made on the procedure as appropriate.

The target study settings were teaching or provincial hospitals from all nine provinces of Sri Lanka. A multi-staged sampling technique was used to select five hospitals from the list. Written permission to conduct the study was obtained from the directors of the hospitals. Verbal consent was obtained from the sisters-in-charge of each unit at the time of assessment. All the units which were designated to perform chest drain insertion at each of the selected hospitals were included. The CTU at THK that was included in the pre-test was excluded from the main study to minimize information bias as data collectors were from that unit.

The study was performed on the designated days by the trained data collectors. Number and percentage calculations of the equipment available within each unit was done.

\section{Results}

Ten surgical instruments were required to be within a unit (table 1) while another nine items were required to be within a sterilized "chest drain pack" (table 2). Twenty nine units were assessed from five tertiary care units in five provinces of Sri Lanka and the availability of each item is indicated in the tables 1 and 2: Although 75.9\% units had designated "chest drain insertion packs", the mean availability of instruments inside them were $52.5 \%$. Only $7 \%$ of units had more than one pack. A tendency not to keep instruments packed away specifically for chest drain insertion was noted, especially in the high output centres where the nurses felt that they could quickly set up a trolley when needed. However, no clear list was available to guide them to do so and some instruments were given only when specifically requested, often one at a time. Of the equipment that were required to be "easily available" within the unit, the availability was $94.8 \%$ units. However, only $24 \%$ of units used the safer non-trocar chest drain exclusively. Furthermore, $27 \%$ of units did not have the curved instrument required for dissection to facilitate safe insertion of this drain.

\section{Discussion}

The first documented description of a closed chest tube drainage system was by Hewett in 1867 for empyema [5]. During the 2nd world war (1939-1945), the experience gained contributed to the development of tube thoracostomy in chest trauma management. By the Vietnam war (1955-1975), chest drains had become the standard of care for management of chest trauma [6]. In 1992, Lilianthal reported the postoperative use of chest tubes following lung resection for suppurative lung diseases [7].

Recently, there has been concern regarding the safety of the insertion of chest drains. Elsayed et al., in their article " Chest drain insertion is not a harmless procedure - are we doing it safely?" concluded that the majority of junior doctors do not have the basic knowledge to insert a chest drain safely and that further training in this procedure is needed for them [8]. In a letter by Hewitt et al. that appeared in the BMJ in 1997, the almost universal lack of standard equipment for chest drain insertion in hospitals was highlighted. The authors suggested that this could be a significant cause of iatrogenic injuries [9].

We found no published guidelines on the equipment requirement for chest drain insertion tailored to the local setting, nor any publications pertaining to the practice of chest drain insertion in Sri Lanka, despite the large number of drains that are inserted throughout the hospitals in our country. This is the first study to address this gap of knowledge in this potentially life-saving procedure. 
Table 1. Items required to be kept within a unit

\begin{tabular}{|c|c|c|c|c|c|c|}
\hline Item & Galle & NHSL & Kandy & A'pura & Jaffna & Total \\
\hline Sterile gown & $6 / 7$ & $6 / 7$ & $3 / 5 \quad 60 \%$ & $1 / 4 \quad 25 \%$ & $5 / 6 \quad 83.3 \%$ & $21 / 2972.4 \%$ \\
\hline Gloves & $7 / 7 \quad 100 \%$ & $7 / 7 \quad 100 \%$ & $5 / 5 \quad 100 \%$ & $4 / 4 \quad 100 \%$ & $6 / 6 \quad 100 \%$ & $29 / 29 \quad 100 \%$ \\
\hline Anti & $7 / 7 \quad 100 \%$ & $7 / 7 \quad 100 \%$ & $5 / 5 \quad 100 \%$ & $4 / 4 \quad 100 \%$ & $6 / 6 \quad 100 \%$ & $29 / 29 \quad 100 \%$ \\
\hline Local anesthetic & $7 / 7 \quad 100 \%$ & $7 / 7 \quad 100 \%$ & $5 / 5 \quad 100 \%$ & $4 / 4 \quad 100 \%$ & $5 / 6 \quad 83.3 \%$ & $28 / 2996.6 \%$ \\
\hline Syringe & $7 / 7 \quad 100 \%$ & $7 / 7 \quad 100 \%$ & $5 / 5 \quad 100 \%$ & $4 / 4 \quad 100 \%$ & $6 / 6 \quad 100 \%$ & $29 / 29 \quad 100 \%$ \\
\hline Blade & $7 / 7 \quad 100 \%$ & $7 / 7 \quad 100 \%$ & $\begin{array}{lll}5 / 5 & 100 \%\end{array}$ & $4 / 4 \quad 100 \%$ & $6 / 6 \quad 100 \%$ & $29 / 29 \quad 100 \%$ \\
\hline Suture (Needle & $7 / 7 \quad 100 \%$ & $7 / 7 \quad 100 \%$ & $4 / 5 \quad 80 \%$ & $3 / 4 \quad 75 \%$ & $5 / 6 \quad 83.3 \%$ & $26 / 29 \quad 89.7 \%$ \\
\hline Chest & $7 / 7 \quad 100 \%$ & $7 / 7 \quad 100 \%$ & $5 / 5 \quad 100 \%$ & $3 / 4 \quad 75 \%$ & $5 / 6 \quad 83.3 \%$ & $27 / 2993.1 \%$ \\
\hline Chest tube- non & $6 / 7$ & $7 / 7 \quad 100 \%$ & $3 / 5 \quad 60 \%$ & $0 / 4$ & $1 / 6 \quad 16.7 \%$ & $7 / 29 \quad 24.1 \%$ \\
\hline $\begin{array}{l}\text { Closed drainage system \& } \\
\text { connecting tubing }\end{array}$ & $7 / 7 \quad 100 \%$ & $7 / 7 \quad 100 \%$ & $5 / 5 \quad 100 \%$ & $4 / 4 \quad 100 \%$ & $6 / 6 \quad 100 \%$ & $29 / 29 \quad 100 \%$ \\
\hline Plaster & $7 / 7100 \%$ & $7 / 7100 \%$ & $5 / 5100 \%$ & $4 / 4100 \%$ & $5 / 683.3 \%$ & $28 / 2996.6 \%$ \\
\hline
\end{tabular}

*Or available as separate eyed-needle and thread in sterile pack

Table 2. Items required to be kept inside a sterile pack

\begin{tabular}{|c|c|c|c|c|c|c|}
\hline Item & Galle & NHSL & Kandy & A'pura & Jaffna & Total \\
\hline Swabs & $5 / 7 \quad 71.4 \%$ & $2 / 7 \quad 28.6 \%$ & $5 / 5 \quad 100 \%$ & $4 / 4 \quad 100 \%$ & $3 / 6 \quad 50 \%$ & $19 / 29 \quad 65.5 \%$ \\
\hline Sponge holder & $1 / 7 \quad 14.3 \%$ & $2 / 7 \quad 28.6 \%$ & $5 / 5 \quad 100 \%$ & $3 / 4 \quad 75 \%$ & $3 / 6 \quad 50 \%$ & $14 / 19 \quad 48.3 \%$ \\
\hline Drapes & $3 / 7 \quad 42.9 \%$ & $3 / 7 \quad 42.9 \%$ & $4 / 5 \quad 80 \%$ & $4 / 4 \quad 100 \%$ & $3 / 6 \quad 50 \%$ & $17 / 29 \quad 58.6 \%$ \\
\hline Towel clips & $1 / 7 \quad 14.3 \%$ & $1 / 7 \quad 14.3 \%$ & $0 \%$ & $0 / 4$ & $1 / 6 \quad 16.7 \%$ & $3 / 29 \quad 10.3 \%$ \\
\hline Scalpel handle & $5 / 7 \quad 71.4 \%$ & $2 / 7 \quad 28.6 \%$ & $5 / 5 \quad 100 \%$ & $4 / 4 \quad 100 \%$ & $2 / 6 \quad 33.3 \%$ & $18 / 29 \quad 62.1 \%$ \\
\hline Needle Holder + Forceps & $2 / 7 \quad 28.6 \%$ & $2 / 7 \quad 28.6 \%$ & $60 \%$ & $2 / 4 \quad 50 \%$ & $50 \%$ & $12 / 29 \quad 41.4 \%$ \\
\hline Dressing scissors & $5 / 7 \quad 71.4 \%$ & $4 / 7 \quad 57.1 \%$ & $5 / 5 \quad 100 \%$ & $4 / 4 \quad 100 \%$ & $4 / 6 \quad 66.7 \%$ & $22 / 29 \quad 75.9 \%$ \\
\hline $\begin{array}{l}\text { Curved instrument for } \\
\text { dissection }\end{array}$ & $4 / 7 \quad 57.1 \%$ & $2 / 7 \quad 28.6 \%$ & $80 \%$ & $75 \%$ & $5 / 6 \quad 83.3 \%$ & $18 / 29 \quad 62.1 \%$ \\
\hline Tubing clamps & $4 / 7 \quad 57.1 \%$ & $0 \%$ & $80 \%$ & $4 / 4 \quad 100 \%$ & $2 / 6 \quad 33.3 \%$ & $14 / 29 \quad 48.3 \%$ \\
\hline
\end{tabular}

Table 3. Check list for equipment needed for chest drain insertion

\begin{tabular}{|l|l|}
\hline Items required to be kept in the unit & $\begin{array}{l}\text { Items required to be kept inside a sterile pack/ } \\
\text { to be set up on a sterile trolley on request }\end{array}$ \\
\hline Sterile gown & Swabs \\
\hline Gloves & Sponge holder \\
\hline Antiseptic solution & Drapes \\
\hline Local anesthetic & Towel clips \\
\hline Syringe and needle & Scalpel handle \\
\hline Blade & Needle holder + forceps \\
\hline Suture (needle + thread)* & Dressing scissors \\
\hline Non trocar chest tube & Curved instrument for dissection \\
\hline Closed drainage system \& connecting tubing & Tubing clamps \\
\hline Plaster & \\
\hline
\end{tabular}

*Or available as separate eyed-needle and thread in sterile pack 
A sterile "chest drain pack" has been traditionally considered a must, as it is an emergency procedure. The present study revealed that although $75.9 \%$ units had so-called chest drain packs, the availability of almost all instruments within them were $<65 \%$. Most of the equipment required to be within the unit were available in $>72.4 \%$ units except for non-trocar chest drains (further discussed below). These factors may lead to delay in performing the procedure and a higher rate of complications. The situation would be worse in the face of multiple casualties as only $7 \%$ had more than one designated pack.

With this back ground, and limited resources, it became evident to the investigators that a reasonable solution to our country would be to make available a list of equipment to be circulated among units so that they may set up a trolley at short notice. In contrast to the costly pre-packed disposable packs used in developed countries, this solution would have the advantage of being more economical.

The situation concerning the actual chest drain itself needs special consideration: In principle, chest drain insertion can be performed by using trocar or non-trocar techniques. In our study $31 \%$ of units had only chest tubes with trocars, while $38 \%$ of units had both trocar and non-trocar chest tubes. Ortener et al. have shown that chest drain insertion with trocars to be associated with a 6-14\% operator related complication rate [3]. No difference in the average performance time could be found between the two techniques. Misplacement and organ injuries occurred more frequently with sharp-tipped trocars. Despite these set-backs it was not clear from this study why our hospitals had more trocar chest drains, especially as they were more expensive. For example, at the time of writing, a size $32 \mathrm{~F}$ trocar chest drain costs LKR 366 for the government while its non-trocar equivalent costs LKR 166. A lack of understanding and motivation of those who place the orders may be the cause.

If a trocar is not used, blunt dissection is essential for inserting a chest drain. In 2012, Kesieme et al. of Nigeria, in a literature survey found that complication rates are increased by the trocar technique [10]. Our study showed $27 \%$ of units had no curved instruments such as Roberts or Sawtell forceps for blunt dissection. This could explain why the operator had to use a trocar tip to facilitate the insertion of the tube. If this was practiced by junior doctors and remained unchecked by their seniors over the years, it is likely that trocar chest drains would have been provided by the nurses and administrators and thus be ordered in large quantities. Sharp tipped-trocars, however, can easily result in iatrogenic complications such as pneumothoraces and visceral injuries. The reason for the lack of curved instruments and non-trocar chest drains are likely to be due lack of knowledge and motivation among doctors and nurses. Making available the above check-list as a guideline is likely to rectify this situation partially. For better results, it will need to be accompanied by an educational program.

The hospitals selected to sample were those thought to have the best facilities from each province. Therefore, the actual situation is likely to be worse than what is seen in this study.

This is the first study that provides objective evidence of the deficiencies pertaining to safe chest drain insertion in Sri Lanka. This is a pragmatic study in that it identifies key areas which will need corrective measures. These include making available the checklist for chest drain insertion (Table 3 ) to the College of Surgeons and the Ministry of Health and via the Ministry, to all health institutions in the country. Furthermore, the Ministry will be requested to consider ordering non-trocar chest drains in light of improving safety and reducing costs.

All authors disclose no conflict of interest. The study was conducted in accordance with the ethical standards of the relevant institutional or national ethics committee and the Helsinki Declaration of 1975, as revised in 2000 .

\section{References}

1. Harris A, O'Driscoll BR, Turkington PM.Survey of major complications of intercostal chest drains insertion in the UK. Postgrad MedJ.2010 Feb; 86(1012):68-72.

https://doi.org/10.1136/pgmj.2009.087759

2. Hyde J, Sykes T, Graham T. Reducing morbidity from chest drains. BMJ 1997; 311: 914-5.

https://doi.org/10.1136/bmj.314.7085.914

3. Ortner CM, Ruetzler K, Schaumann N, Lorenz V, Schellongowski P, Schuster E, Salem RM, Frass M. Evaluation of performance of two different chest tubes with either a sharp or a blunt tip for thoracostomy in 100 human cadavers. Scand $J$ Trauma Resusc Emerg Med. 2012 Feb 2; 20:10. https://doi.org/10.1186/1757-7241-20-10

4. D Laws, E Neville, J Duffy, on behalf of the British Thoracic Society Pleural Disease Group, a subgroup of the British Thoracic Society Standards of Care Committee. BTS guidelines for the insertion of a chest drain. Thorax 2003;58 (Suppl II):ii53-ii59. https://doi.org/10.1136/thx.58.suppl_2.ii53

5. Hewett FC. Thoracentesis: the plan of continuous aspiration. The British Medical Journal.1876;1 (793):p. 317. https://doi.org/10.1136/bmj.1.793.317

6. Monaghan SF, Swan KG. Tube thoracostomy: the struggle to the 'standard of care' Annals of Thoracic Surgery. 2008;86 (6):2019-2022. https://doi.org/10.1016/j.athoracsur.2008.08.006

7. Lilienthal $\mathrm{H}$. Resection of the lung for suppurative infections with a report based on 31 operative cases in which resection was done or intended. Annals of Surgery. 1922;75(3):257-320 https://doi.org/10.1097/00000658-192203000-00001

8. Elsayed H, Roberts R, Emadi M, Whittle I, Shackcloth M. Chest drain insertion is not a harmless procedure--are we doing it safely? Interact Cardiovasc Thorac Surg. 2010 Dec;11(6):745-8. https://doi.org/10.1510/icvts.2010.243196

9. Hewett FC. Thoracocentesis: the plan of continous aspiration . The British Medical Journal.1876;1(793):p 217

https://doi.org/10.1136/bmj.1.793.317

10. Kesieme EB, Dongo A, Ezemba N, Irekpita E, Jebbin N, Kesieme C. Tube thoracostomy: complications and its management. Pulm Med 2012;2012:256878. Epub 2011 Oct 16. 